\title{
LOW SIGNAL FEL GAIN: MEASUREMENT, SIMULATION AND ANALYSIS*
}

\author{
R. Schoenlein ${ }^{\dagger}$, H. Chong, T.E. Glover, P. Heimann, A. Zholents, M. Zolotorev \\ LBNL, Berkeley, CA 94720
}

\begin{abstract}
We describe an experimental set-up and measurement technique for measurement of the FEL gain at the Advanced Light Source. Measurement results are compared with computer simulations and analitical expression that includes effects of the laser beam difraction and finite electron beam size.
\end{abstract}

\section{INTRODUCTION}

This work is a part of a broader research effort to develop a source of femtosecond $\mathrm{x}$-ray pulses based on an electron storage ring [1]. The technique that we are currently using at the ALS is based on energy modulation of the electrons as they traverse the wiggler with a co-propagating femtosecond laser pulse [2]. The optimal interaction of the electrons with the electric field in the laser pulse occurs when spontaneous electron radiation in the wiggler overlaps with the field of laser radiation in the transverse phase space, and when frequencies and spectral bandwidths of the electron emission and laser radiation are matched. The same conditions are required for achieving the maximum gain in the laser intensity, except the laser frequency is shifted to the point where the derivative of the spectral function of the electron spontaneous emission in the wiggler has a maximum (Madey's theorem [3]). Therefore, we determine the amplitude of the laser/e-beam energy modulation by measuring the gain in the laser intensity and use this as a diagnostic tool for a fine tuning of the experimental conditions.

\section{MEASUREMENTS}

Measurements of the gain of the laser pulse intensity for a laser pulse co-propagating with an electron bunch in the wiggler magnet have been performed at the Advanced Light Source. We use the electron beam at $1.5 \mathrm{GeV}$ with horizontal and vertical beam emittances of $4 \times 10^{-7} \mathrm{~cm}$ and $1 \times 10^{-8} \mathrm{~cm}$ respectively and energy spread of $8 \times 10^{-4}$. The wiggler magnet has a period $\lambda_{w}=16 \mathrm{~cm}, 19$ periods and its gap is adjusted to allow first harmonic electron emission at $\sim 800 \mathrm{~nm}$ (wiggler deflection parameter $a_{w} \simeq 13$ ). A schematic of the experimental set up is shown in Figure 1.

The laser system consists of a Ti:Sapphire laser oscillator, stretcher, amplifier, and compressor. It produces $\sim 0.5$ $\mathrm{mJ}, \sim 70$ fs laser pulses at $1 \mathrm{kHz}$ repetition rate. The laser oscillator is synchronized to the storage ring RF mas-

\footnotetext{
* Work supported by DoE under contract No DE-AC03-76SF00098.

† Email: RWSchoenlein@lbl.gov
}

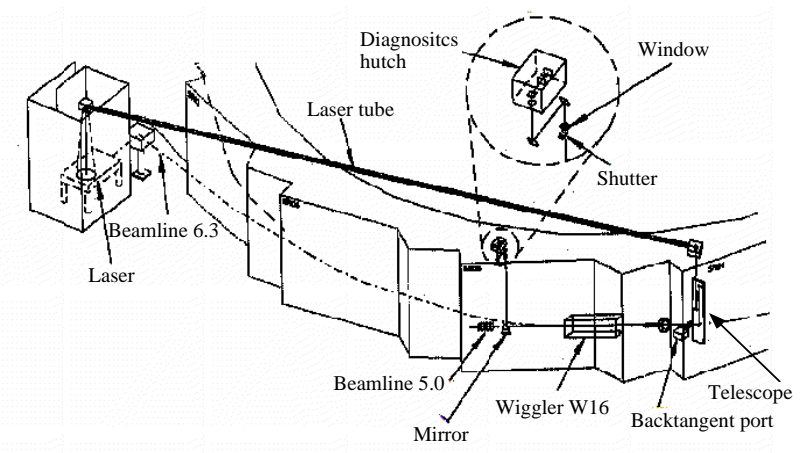

Figure 1: The layout of the experiment.

ter oscillator. The amplified Ti:Sapphire laser pulses enter the main vacuum chamber through a back-tangent window in the vacuum chamber of the wiggler insertion and co-propagate with the electron beam through wiggler. A mirror following the wiggler reflects the laser light and the electron wiggler emission out of the vacuum chamber for diagnostic purposes. Images of the near field and far field wiggler radiation are observed on a CCD camera, and the near and far field modes of the laser propagating through the wiggler are matched to the wiggler radiation using a remotely adjustable telescope at the back tangent port. A band-pass interference filter with $\sim 40 \mathrm{~nm}$ bandwidth at 800 $\mathrm{nm}$ is inserted in front of the CCD in order to discriminate the higher-order harmonics. Spectra are measured using

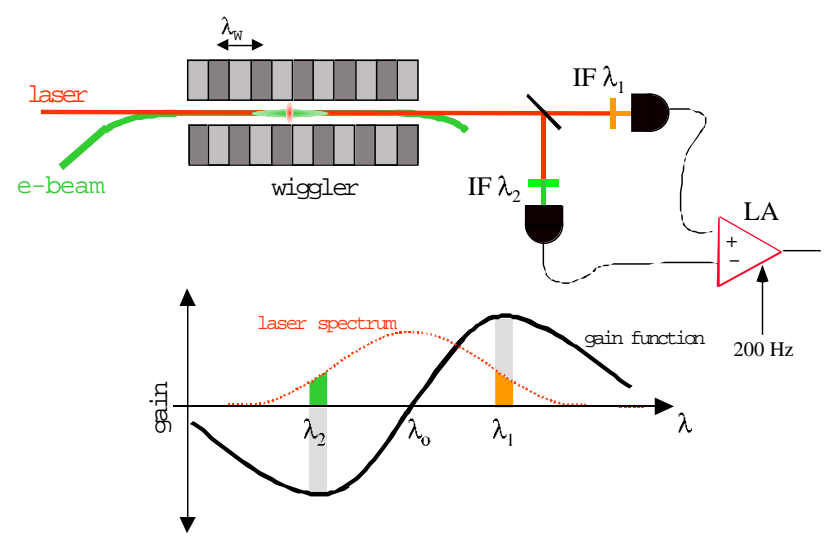

Figure 2: A schematic of the gain measurement (IF - interference filter, LA - lock-in amplifier).

a spectrometer. Temporal overlap between the laser pulse and a single electron bunch is accomplished by monitoring both pulses using a high-speed ( $\sim 100$ ps FWHM) photodi- 
ode, and adjusting the path delay of the laser pulse.

Laser gain is measured with the following technique. Two interference filters with bandwidth $\sim 10 \mathrm{~nm}$ are used to select two portions of the broadband laser spectrum which are near the maximum and minimum of the gain curve at $\sim 815 \mathrm{~nm}$ and $\sim 785 \mathrm{~nm}$ (see, Figure 2). This technique provides the additional advantage of differential detection, which effectively doubles the measured signal, and eliminates noise due to laser power fluctuations. The observed gain is modulated on and off by shifting the laser pulse timing by $14 \mathrm{~ns}$ (a round trip time in the laser oscillator) to be coincident and anti-coincident with the electron bunch at a modulation frequency of $\sim 200 \mathrm{~Hz}$. This allows for phase-

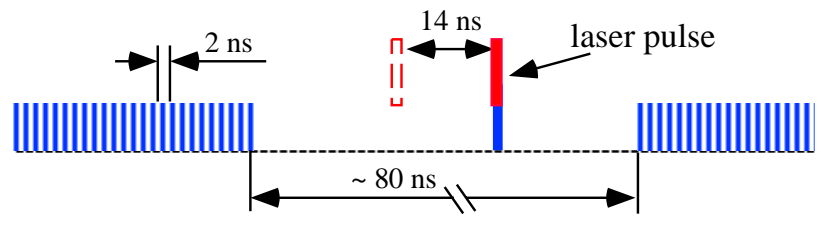

Figure 3: A schematic of the gain modulation.

locked detection of the laser gain. During multibunch operation the measurement is conducted with a single electron bunch positioned in the gap of the bunch train as shown in Figure 3. A result of the gain measurement is shown in Figure 4. During this measurement a short $\sim 70$ fs laser pulse is scanned in time through the electron bunch thus revealing the electron bunch longitudinal structure. We show in the next section that the gain is proportional to the electron peak current.

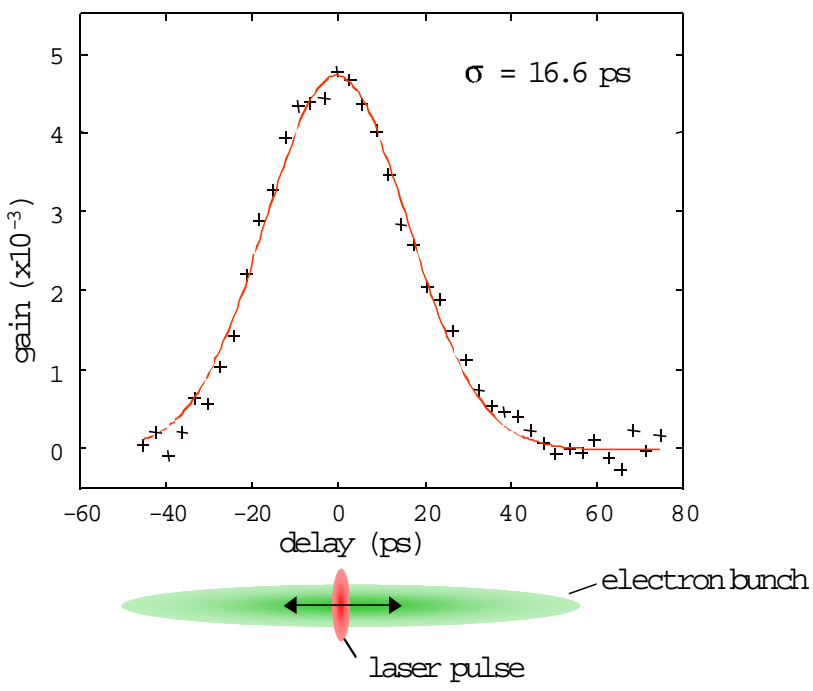

Figure 4: Gain measurement at different time delays of the femtosecond laser pulse.

\section{ANALYSIS}

An expression for the small signal gain of the laser spectral intensity in the FEL including effects of the laser beam diffraction and finite electron beam size has previously been found in [5]. Re-written for a planar wiggler and for arbitrary wiggler deflection parameter $a_{w}$ it is:

$$
g\left(\omega, \sigma_{\perp}, Z_{R}\right)=-4 \frac{a_{w}^{2} / 2}{1+a_{w}^{2} / 2}(2 \pi M)^{2} \frac{I}{\gamma I_{A}} f(\nu, \xi, q),
$$

where $\gamma$ is the Lorentz factor, $I$ is the electron peak current, $I_{A} \simeq 17 \mathrm{kA}$ is the Alfvén current, $M$ is the number of wiggler periods, and the functional dependence of $g$ on frequency $\omega$, electron beam size in the wiggler $\sigma_{\perp}$, and Rayleigh length $Z_{R}$, is described by the gain function $f(\nu, \xi, q)$ with variables $q=L / Z_{R}$, where $L$ is the wiggler length, $\xi=k \sigma_{\perp}^{2} / Z_{R}$, where $k$ is the laser wave number, and $\nu=2 \pi M \frac{\omega-\omega_{e}}{\omega_{e}}$, where $\omega_{e}=\frac{2 \pi c}{\lambda_{w}} \frac{2 \gamma^{2}}{1+a_{w}^{2} / 2}$ is the frequency of the electron wiggler emission and $c$ is the speed of light:

$$
f(\nu, \xi, q)=\operatorname{Re} \frac{d}{d \nu} \int_{0.5}^{0.5} d \tau \int_{0.5}^{\tau^{\prime}} d \tau^{\prime} \times
$$

$$
\frac{q \exp \left\{-i \nu\left(\tau-\tau^{\prime}\right)\right\}}{\left(1+i q \tau^{\prime}\right)(1-i q \tau)+\xi\left(2+i q \tau^{\prime}-i q \tau\right)},
$$

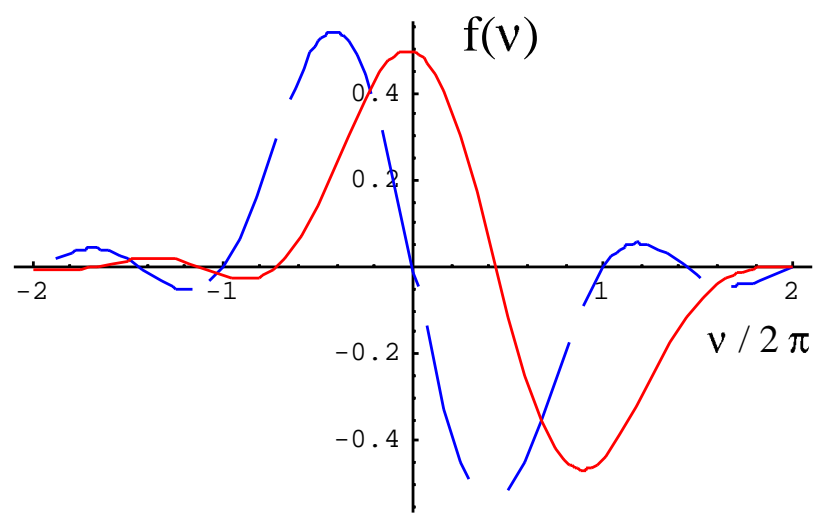

Figure 5: Gain function $f(\nu, \xi, q)$ calculated at $\xi=0$ and $q=4$ (red curve). Dashed blue curve is the gain function in one dimensional approximation.

The function $f(\nu, \xi, q)$ calculated at $q=4$ (shown to be an optimal choice for a maximum gain [5]) and $\xi=0$ is plotted in Figure 5. For a comparison we also plot the gain function $d / d(\nu / 2)[2 \sin (\nu / 2) / \nu]^{2}$ [3] that characterizes the small signal FEL gain for a 1D case (ignoring laser beam diffraction and finite electron beam size). The main difference of the gain function (2) from the 1D case consists of a spectral shift. It can be understood by recalling that formula (2) is written for the $3 \mathrm{D}$ case in which the laser beam is focused in the wiggler. The phase velocity of the laser field in the focus is greater than velocity of the light in vacuum (this effect is known as a Guoy phase shift). Therefore, in order to maintain optimal interaction with electrons over the entire wiggler length the laser frequency must be red shifted relative to the maximum frequency of the electron wiggler emission. 
The same phenomena of the spectral shift can be equivalently explained by the angular-frequency correlation in the electron wiggler emission $\omega_{e}(\theta)=$ $\omega_{e}(0)\left\{1+\frac{\gamma^{2} \theta^{2}}{\left(1+a_{w}^{2} / 2\right)}\right\}^{-1}$, where $\theta$ is the observation angle. This correlation causes that the maximum spectral intensity of the electron wiggler emission integrated over the solid angle is the red shifted relative to $\omega_{e}(0)$. Therefore, for a better matching of the fields of the laser radiation and the electron wiggler emission in the far field region, the laser frequency must be red shifted by the same amount.

In the experiment we have $L=3 \mathrm{~m}, Z_{R} \simeq L / 4$, and a beta function in the wiggler of $\beta \simeq 11 \mathrm{~m}$. With this beta function the angular divergence of the electron beam in the wiggler is negligible. Thus, Eq.(1) that accounts only for a finite beam size, correctly describes our experiment. Figure 6 shows the dependence of the gain on $\xi$. Calculation usings Eq.(1) is shown with red circles. For each $\xi$ value the gain was taken at a position of the maximum of the gain function $f(\nu, \xi, q=4)$. The result of computer simulations using GINGER [4] is shown with blue rhombs. The nominal operation condition corresponds to $\xi=0.46$. A solid line in Figure 6 is a fit of the Eq.(1) using the following dependence of the gain on the electron beam size:

$$
g\left(\omega, \sigma_{\perp}, Z_{R}\right)=g\left(\omega, \sigma_{\perp}=0, Z_{R}\right) /(1+\xi) .
$$

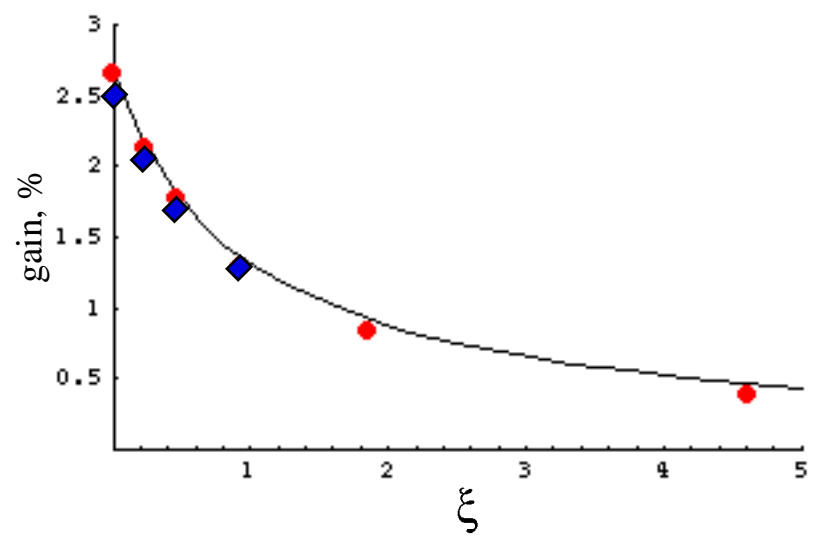

Figure 6: Gain versus $\xi$. Solid line is a fit following Eq.(3). Red circles are numerical calculations using Eq.(1). Blue rhombs are GINGER simulations. The rhomb and the circle overlap at $\xi=0.92$.

At the time of writing we performed $\sim 50$ measurements of the FEL gain. Typically, measured gains are reproducible over period of $\sim 8$ hours, but vary somewhat on a day to day basis. Some days we measure $\sim 60 \%$ of the predicted value and some days $\sim 40 \%$. The discrepancy may be related to the imperfections in the mode structure of the laser light, which we do not test routinely. It seems less likely that the observed losses can be explained by seismic vibrations of the supporting structures for the optics or by air turbulence in the laser beam path.

\section{CONCLUSION}

Preliminary results of the FEL gain measurements at the ALS show that these measurements can be used for fine tuning of the efficiency of the laser/e-beam interaction in the wiggler. We found that the gain is a very sensitive indicator of any deviation from optimal interaction conditions. So far the measured gain disagrees with the predicted one in the analytical calculations giving $\sim 40-60 \%$ of the expected value. The reasons for this discrepancy are being studied.

Acknowledgement. The authors wish to aknowledge the technical support of the LBNL laser safety oficer K. Barat and the staff of the ALS in setting-up this experiment.

\section{REFERENCES}

[1] A. Zholents, et. al. in Proc. of this Conf., report WEP44.

[2] A. Zholents and M. Zolotorev, PRL v.76, (1996)912.

[3] J.M.J. Madey, Nuovo Cimento 50B, (1979)64.

[4] W. Fawley, GINGER documentation, unpublished.

[5] A. Amir, Y. Greenzweig, NIM A250, (1986)404. 